\title{
Experimental investigation of mode I fracture for brittle tube-shaped particles
}

\author{
Marta Stasiak ${ }^{1,2, \star}$, Gaël Combe ${ }^{1,2}$, Jacques Desrues ${ }^{1,2}$, Vincent Richefeu ${ }^{1,2}$, Pascal Villard $^{1,2}$, Gilles Armand ${ }^{3}$, and \\ Jad Zghondi ${ }^{3}$ \\ ${ }^{1}$ Univ. Grenoble Alpes, 3SR, F-38000 Grenoble, France \\ ${ }^{2}$ CNRS, 3SR, F-38000 Grenoble, France \\ ${ }^{3}$ Andra, R\&D Division, Meuse/Haute-Marne Underground Research Laboratory, 55290 Bure, France
}

\begin{abstract}
We focus herein on the mechanical behavior of highly crushable grains. The object of our interest, named shell, is a hollow cylinder grain with ring cross-section, made of baked clay. The objective is to model the fragmentation of such shells, by means of discrete element (DE) approach. To this end, fracture modes $I$ (opening fracture) and $I I$ (in-plane shear fracture) have to be investigated experimentally. This paper is essentially dedicated to mode $I$ fracture. Therefore, a campaign of Brazilian-like compression tests, that result in crack opening, has been performed. The distribution of the occurrence of tensile strength is shown to obey a Weibull distribution for the studied shells, and Weibull's modulus was quantified. Finally, an estimate of the numerical/physical parameters required in a DE model (local strength), is proposed on the basis of the energy required to fracture through a given surface in mode $I$ or $I I$.
\end{abstract}

\section{Introduction}

The discrete element method (DEM) is commonly used to model the response of rigid grains assemblies [1-3], but it has been also employed by various researchers in studying the behavior of crushable particles packings $[4,5]$.

Among all existing approaches capable of modeling particle breakage, two are frequently used. The first one takes into consideration the rigid particles that will be replaced by smaller ones, when the breakage must occur (according to the grain loading criterion) [6]. This approach has the advantage of the simplicity but may have the disadvantage of having material losses under certain conditions. In the second approach the grain is created by assembling smaller particles together $[7,8]$ - then the assembly bonds correspond to the pre-cracks of the grain. It is this second approach that we will choose in this article.

In this study, we focus on the peculiar 3D shape of grain which is a cylinder with ring cross-section, referred to as shell. Whereas we aim to study the micro-macro mechanical behavior of large, dense assemblies of crushable shells, we propose herein a strategy to model 3D grains, shaped as hollow cylinders, by means of DEM.

Since the packing of such shells is a new granular material, this research starts, at the grain scale, with the investigation of a single shell fracturing. Firstly, an experimental campaign has been performed, in order to extract the actual strength of the shells. Since these shells are made of baked clay, it is worth noting that the strength of brittle material may obey Weibull statistics, based on the theory of the weakest link. The distribution of the tensile strength,

^e-mail: marta.stasiak@grenoble-3sr.fr that prevents mode I fracture, is then analyzed assuming that it is related to the shell geometry. With the objective to model the shell fracture with a discrete element approach, an estimate of the DEM strength-parameters is proposed for the cluster of sphero-polyhedra (the shell is subdivided into parts bonded together by means of a force-law).

\section{Mechanical properties of the shells}

The particles, made of baked clay, are tube-shaped shells (Figure 1(a)) and result from an industrial process. In fact, two grain sizes are manufactured. Before been baked at high temperature, the particle diameter is either 18 or 20 $\mathrm{mm}$. Once baked, the grain dimensions, sketched in Figure 1(a), show small geometrical variations as reported in Table 1.

A number of radial compression tests (Brazilian-like tests) have been performed (see inset in Figure 2(a)). This test has been chosen because it allows indirect measurement of a tensile strength and involves crack opening, that corresponds to mode $I$ fracture. These experiments have

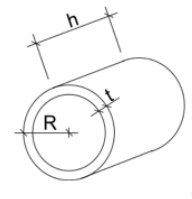

(a)
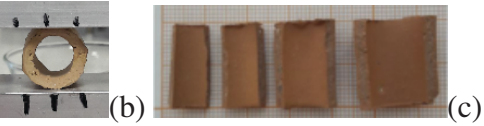

Figure 1: Studied shell: (a) its dimensions, (b) position in the experimental setup, and (c) four pieces after breakage. 
Table 1: Name of the size, number of tests and geometrical description of tested tube-shaped shells. See Figure 1(a).

\begin{tabular}{ccccc}
\hline & No. & $D=2 R[\mathrm{~mm}]$ & $h[\mathrm{~mm}]$ & $t[\mathrm{~mm}]$ \\
\cline { 2 - 5 } D18 & 33 & $16.5 \pm 0.4$ & $17.4 \pm 1.3$ & $2.4 \pm 0.1$ \\
D20 & 50 & $18.2 \pm 0.4$ & $18.4 \pm 1.5$ & $2.8 \pm 0.1$ \\
\hline
\end{tabular}

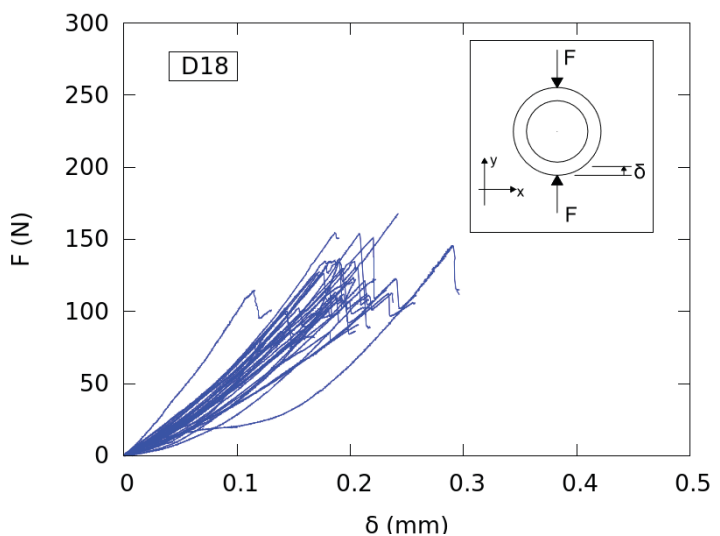

(a)

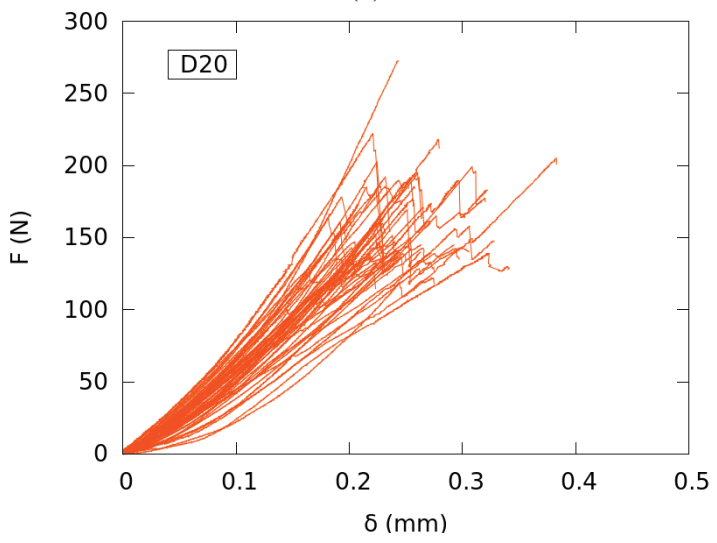

(b)

Figure 2: Force $F v s$. vertical displacement $\delta$ curves resulting from the radial compression tests, performed on two sizes of tube-shaped grains with a constant imposed displacement rate $(\dot{\delta}=0.02 \mathrm{~mm} / \mathrm{s})$ : (a) D18 and (b) D20. Inset: a sketch of the loading condition.

been performed using a standard loading frame. A vertical force $F$ was measured on the outside wall of the shell; Figure 1(b). The total number of performed tests is given in Table 1. Each test ended when the shell broke most often into four pieces as shown in Figure 1(c). The radial shell shortening $\delta$ and the vertical compressive force $F$ were recorded all along each test. Figures 2(a) and 2(b) show all force-displacement curves for tested shells D18 and D20, respectively.

From the compression tests, two quantities can be extracted: the maximum force at failure $F_{\max }$ and the corresponding vertical shortening $\delta_{\max }$ of the shell. Observed variations in the mechanical response of the shells, as shown in Figure 2, may result from the geometrical heterogeneity of the particles (see Table 1), but also from the material heterogeneity. In particular, some internal flaws such as air bubbles can be trapped in the crude clay when it is formed.

When failure arises, the maximum tensile stress is localized at the vertical of the compression force on the inner cylindrical wall of the shell, as illustrated in Figure 3. For this configuration, it can be shown that $\sigma_{\max }$ is a function of the external radius $R$, the thickness $t$ and the height $h$ of the shell [11]. Assuming that the thickness $t$ does not vary significantly from one shell to another, it is shown that

$$
\sigma_{\max } \propto \frac{F_{\max }}{R h} \mathcal{K}(t),
$$

where $\mathcal{K}(t)$ is a stress concentration factor that depends on $t$. Thanks to its very small variation from a shell to another, $t$ is hereafter (and consequently $\mathcal{K}(t)$ ) assumed to be constant. Figure 4 reports the forces $F_{\max }$ as a function of the vertical shortening $\delta_{\max }$ at breakage. In the inset of the same figure, $F_{\max }$ has been divided by $R h$ to scale with the maximum tensile stress at breakage, and $\delta_{\max }$ has been divided by $R$ to scale with the radial strain. That way, it is expected to cancel the scattering due to geometrical differences between the shells. However, no collapse is observed even if the cloud of points seems to be less spread. The variations in strength is thus a consequence of the heterogeneity of the clay matter itself.

\section{Statistical analysis of tensile strength}

The Weibull theory is supposed to be adequate for describing the statistical distribution of strength for quasi-brittle material. If $P(x)$ represents the cumulative distribution function of the occurrence of a strength $x$ (this strength can be expressed in terms of force, stress or energy for example), then the survival probability $P_{S}(x)=1-P(x)$ is expressed as follows:

$$
P_{S}(x)=\exp \left\{\left(-\frac{x}{x_{0}}\right)^{m}\right\}
$$

where $m$ is a shape parameter (also called Weibull's modulus) and $x_{0}$ a scale parameter. Note that the higher is the shape parameter $m$, the smaller is the variation of strength $x$. It is convenient to use double logarithmic scale, that results from a linearization of Equation (2), so that the shape

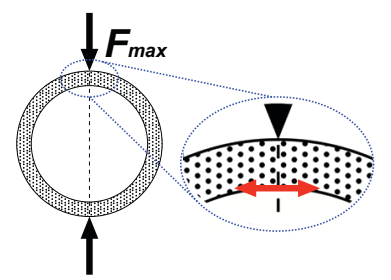

Figure 3: The maximum tensile stress is localized in the direction of the force on the inner wall of the shell. It is here shown by the double red arrows. 


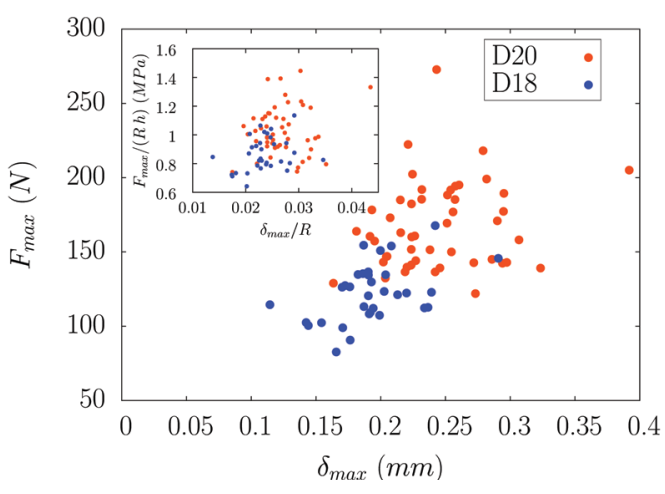

Figure 4: $F_{\max }$ at failure versus the corresponding displacement $\delta_{\max }$ for two grain sizes before normalization. Inset: Maximum force at failure $F_{\max }$ normalized by the radius and the length of the grain versus $\delta_{\max }$ normalized by the radius of the particles.

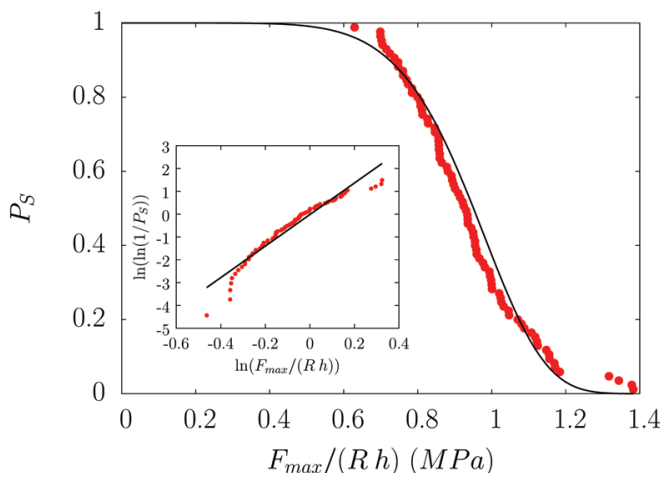

Figure 5: Weibull's distribution of the shell strength. Symbols are experimental data, and continuous line is related to Equation (2). Inset: the same plot in linearized scale Equation (3) - used to identify Weibull's modulus $m=6.7$. For three point bend test, in which the material fails also in tension, various types of ceramics have wide distributions described by Weibull's modulus ranging from 6 to 12 .

parameter can be determined from the slope of this relation:

$$
\ln \circ \ln \left(\frac{1}{P_{S}(x)}\right)=m\left(\ln x-\ln x_{0}\right)
$$

This analysis can be applied with the normal strength $F_{\max }$ measured for the shells, normalizing it with $R h$ to limit the scattering caused by geometric variations. Figure 5(inset) clearly shows that most of the data follow a straight line according to Equation (3), and it obeys, as expected for quasi-brittle materials, the weakest link theory. Hence, Weibull distribution provides here a reliable first order statistical model for setting the shells strength (Figure 5) when submitted to vertical compression.

\section{Towards a DE model for shell crushing}

One final objective of this study is to explore the mechanical response of an assembly of breakable shells by using the DEM. We hope to determine the most relevant geometrical parameters that may influence significantly the shear resistance of such assemblies. Thus, a model of shell needs to be designed for this purpose.

Within an assembly, the loading mechanism of a single shell can be complex, and both mode $I$ and mode $I I$ fractures can be activated. In case of mode $I$, it has been experimentally observed that the fracture surfaces are always radial (Figure 1(c)). Mode II characterization also requires breakage trough radial planes. Therefore, it was chosen to pre-define these surfaces by "sticking" some rigid, polyhedral elements in such a manner that they form the global shape of a shell (Figure 6 (a)). From the DE technical point of view, the adjacent elements are joined via four points, i.e., spheres in contact. To define the contact forces, two directions are considered: the direction I normal to the predefined plan of fracture (Figure 6(b)) and the sliding direction II (Figure 6(c)). The corresponding elastic forces $f_{I}$ and $f_{I I}$ are functions of the relative displacements $\left(d_{I}\right.$ and $d_{I I}$ ) and the respective stiffnesses $k_{I}$ and $k_{I I}$. The behavior of the shell is in the elastic phase, until the following failure surface is reached:

$$
\frac{f_{I}}{f_{I}^{\star}}+\left(\frac{f_{I I}}{f_{I I}^{\star}}\right)^{q}-1=0
$$

where $f_{I}^{\star}$ and $f_{I I}^{\star}$ are the yield cohesive forces for pure tensile and pure shear loading, respectively, and $q$ is a parameter that shape the yield surface.

To complete the particle model, the threshold values $f_{I}^{\star}$ and $f_{I I}^{\star}$ as well as the stiffnesses $k_{I}$ and $k_{I I}$ have to be determined. To this end, one can use the energy storage capacity $W^{\star}$, that for the selected mode of loading can be defined from the experimental force-displacement curve:

$$
\mathcal{W}_{\exp }(\delta)=\int_{0}^{\delta} F \mathrm{~d} \delta
$$

so that $\mathcal{W}^{\star}=\mathcal{W}_{\exp }\left(\delta_{\max }\right)$. Note that $\mathcal{W}_{\exp }(\delta)$ includes an energy contribution, related to shell-device contact, however, it is negligibly small and omitted in modeling concept. From the DEM side, this quantity can be assessed

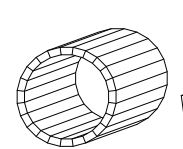

(a)

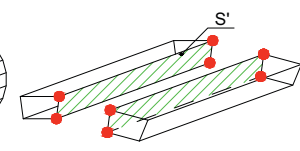

(b)

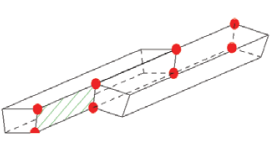

(c)
Figure 6: (a) 3D DEM model of a shell is a cluster of 24 non-deformable sphero-polyhedra. One spheropolyhedron consists of 6 cylinders as edges, 8 spheres as corners and 6 polygons as faces. The adjacent parts are joined via four contact points between the spheres. (b) contact opening in fracture mode $I$, (c) contact opening in fracture mode $I I$ 
by means of the local force-displacement evolution of the bonded links. Assuming $k_{I I}=\frac{2-2 v}{2-v} k_{I}=\beta k_{I}$ [12], where $v$ is the Poisson coefficient, the elastic energy storage reads:

$$
\mathcal{W}_{\mathrm{DEM}}\left(\delta ; k_{I}\right)=\frac{k_{I}}{2} \sum_{\text {contact } c}\left(\left(d_{I}^{(c)}\right)^{2}+\beta\left(d_{I I}^{(c)}\right)^{2}\right)
$$

The value of $k_{I}$ can easily be determined using a single DEM simulation for an arbitrary stiffness $\tilde{k}_{I}$, so that following relation is satisfied:

$$
\frac{k_{I}}{\tilde{k}_{I}}=\frac{W_{\mathrm{exp}}(\delta)}{W_{\mathrm{DEM}}\left(\delta ; \tilde{k}_{I}\right)}
$$

Depending on the main fracture mode involved in the experiment, a first-order estimate of the yield force can be proposed by considering the energy storage capacity per unit surface $W^{\star} / S$, and by noting that each bond withstands a quarter of predefined surface of rupture $S^{\prime}=t h$ :

$$
\left\langle\frac{W_{\bullet}^{\star}}{S}\right\rangle \asymp \frac{4\left\langle f_{\bullet}^{\star}\right\rangle^{2}}{k_{\bullet} t h} \quad \text { thus }\left\langle f_{\bullet}^{\star}\right\rangle \asymp \sqrt{\left\langle\frac{W_{\bullet}^{\star}}{S}\right\rangle \frac{k_{\bullet} t h}{4}}
$$

where $S$ is the total fracture surface; $\langle x\rangle$ is the mean of $x$; the symbol $\bullet$ stands for $I$ or $I I$ depending on the main fracture mode; and the symbol $\asymp$ means that the left and right hand of the expression should evolve similarly and have a comparable order of magnitude.

To perform a simulation of an assembly, the variation of shell strength can be estimated from the distribution of the yield force. Such distribution can be assessed from the distribution of $W^{\star} / S$, and Equation (8) suggests that:

$$
P_{S}\left(f_{I}^{\star}\right) \propto P_{S}\left(\sqrt{W_{I}^{\star} / S}\right)
$$

For the shells, that are made of quasi-brittle material, a Weibull distribution is still expected, and Figure 7 shows such occurrence. It allows us to access the Weibull's modulus for the distribution of $\sqrt{W_{I}^{\star} / S}$ and thus the distribution of $f_{I}^{\star}$ can be quantified.

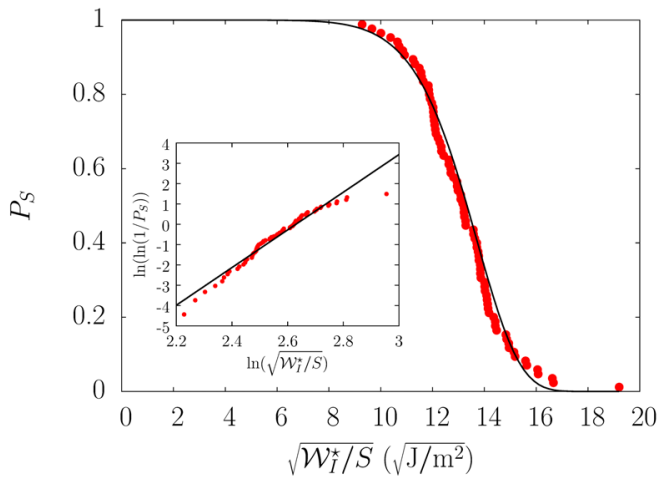

Figure 7: Weibull distribution of the square root of energy storage capacity per unit surface from radial compression tests, that reflects the distribution of the local yielding force for mode $I$ rupture. Symbols are experimental data, and continuous line is related to Equation (2). Inset: the same plot with linearized scale - according to Equation (3) - used to identify Weibull's modulus $m=9.26$

\section{Conclusions and perspectives}

An experimental campaign involving a great number of radial compression tests has been performed. The objective was to characterize the material resistance of tube-shaped grains made of baked clay, called shells. The variability of the macroscopic strength was studied within a statistical framework and it was shown to obey the Weibull theory. To model breakable shells by 3D-DEM, predefined fracture plans are bonded by cohesive elastic forces that can break according to a local yield criterion. A solution to assess experimentally the mean local strengths relies on the macroscopic energy storage capacity for each fracturing mode of the shell. Furthermore, the dispersion of these strengths can be characterized from macroscopic tests, and for the breakage in mode $I$ it is shown to also obey a Weibull distribution.

The experimental campaign needs to be completed with shear tests on the shells to investigate their fracture in mode II. Once a single shell will be satisfactorily modeled, the next step will focus on the mechanical responses of an assembly of breakable shells subjected to triaxial and odometric loadings.

\section{Acknowledgments}

The Laboratoire 3SR is part of the LabEx Tec 21 (Investissements d'Avenir, Grant Agreement No. ANR-11LABX-0030)

\section{References}

[1] CEGEO, B. Saint-Cyr, K. Szarf, C. Voivret, E. Azéma, V. Richefeu, J.-Y. Delenne, G. Combe, C. NouguierLehon, P. Villard, EPL 98(4), 44008 (2012)

[2] Roux J.-N. and Combe G., UTAM-ISIMM Symposium on Mathematical Modeling and Physical Instances of Granular Flows, 1227, 260-270 (2010)

[3] G. Mollon, V. Richefeu, P. Villard, D. Daudon, Journal of Geophysical Research 117, F02036 (2012)

[4] G. R. McDowell \& O. Harireche, Geotechnique 52, 131-135 (2002)

[5] Y. P. Cheng, Y. Nakata \& M. D. Bolton, Geotechnique 53, 633-641 (2003)

[6] D. Cantor, N. Estrada \& E. Azéma, Geomechanics from Micro to Macro (Taylor \& Francis Group, London 2015) 257-262

[7] M. Renouf, B. Gezahengn, M. Abbas \& F. Bourgeois, Powders and Grains 2013 1542, 907 (2013)

[8] A. Aminzadeh \& E. S. Hosseininia, Powders and Grains 2013 1542, 915 (2013)

[9] W. Weibull, ASME Journal of Applied Mechanics 8, 293-297 (1951)

[10] M. Mellor, I. Hawkes, Eng. Geol. 5, 173-225 (1971)

[11] De-Li Ma, Int.J.Pres.Ves.E Piping 42, 185-191 (1990)

[12] I. Agnolin and J.-N. Roux, Phys. Rev. E 76, 061304 